\title{
Doppler-free saturation spectroscopy of polyatomic molecules: Photochemical hole burning of gas phase $s$-tetrazine
}

\author{
A. Kiermeier, K. Dietrich, E. Riedle, and H. J. Neusser \\ Institut für Physikalische und Theoretische Chemie der Technischen Universität München, Lichtenbergstr. \\ 4, D-8046 Garching, West Germany
}

(Received 1 August 1986; accepted 26 September 1986)

\begin{abstract}
Doppler-free spectra of two vibronic bands in the $\tilde{A}\left({ }^{1} B_{3 u}\right)-\tilde{X}\left({ }^{1} A_{g}\right)$ transition of the photochemically instable $s$-tetrazine $\left(\mathrm{H}_{2} \mathrm{C}_{2} \mathrm{~N}_{4}\right)$ are presented. For the first time saturation spectroscopy is successfully applied to a large polyatomic molecule. Photochemical decomposition of $s$-tetrazine molecules takes place after excitation and prevents the molecule from returning to the ground state by radiation and nonradiative processes. This represents a particular type of hole burning in the ground state velocity distribution. The elimination of the inhomogeneous Doppler broadening enables us to determine collisionless homogeneous linewidths. For the $0-0$ band a value of $\gamma=190 \mathrm{MHz}$ is found whereas the $16 a_{1}^{1}$ band at higher excess energy $\left(\nu_{16 a}^{\prime}=256 \mathrm{~cm}^{-1}\right)$ shows a sharper linewidth of $\gamma=140 \mathrm{MHz}$. No rotational dependence of the linewidth is found in the $0-0$ band up to $J$ levels higher than 70. It is argued that internal conversion to the $\tilde{X}\left({ }^{1} A_{g}\right)$ state is the process responsible for the observed linewidths and that dissociation takes place on the electronic ground state potential surface.
\end{abstract}

\section{INTRODUCTION}

Recently we have shown that Doppler-free techniques yield important spectroscopic information on intramolecular interactions within polyatomic molecules. ${ }^{1,2}$ After elimination of the Doppler broadening in the gas phase, the observation of individual quantum states was shown to be possible even in larger polyatomic systems. Doppler-free two-photon measurements of the prototype system benzene revealed rotational line splittings of several $100 \mathrm{MHz}$ which have been attributed to the interaction of the excited light zero order state with a dark background state. ${ }^{1}$ Since the inhomogeneous Doppler broadening is removed in a Doppler-free experiment, homogeneous linewidths have been observed which directly provide information on intramolecular relaxation processes. ${ }^{3}$

Even though there exist several Doppler-free methods, ${ }^{4}$ only Doppler-free two-photon absorption has so far been successfully applied to high resolution studies of large polyatomic molecules in a room temperature thermal distribution. In symmetric molecules, the selection rules for twophoton absorption differ from those of one-photon absorption. ${ }^{5}$ For this reason, the molecular vibronic states which can be studied by two-photon absorption cannot be excited by one-photon absorption and vice versa. In order to obtain complete information on the intramolecular dynamics, high resolution spectra of one-photon states are desirable in some cases.

Saturation and polarization spectroscopy are the onephoton excitation techniques which can lead to an elimination of the Doppler broadening. They have first been demonstrated for atoms ${ }^{6}$ and since that time the techniques have been applied to diatomic ${ }^{7}$ and small polyatomic molecules. ${ }^{8}$

In this work, a Doppler-free saturation spectrum is obtained for the first time for a large polyatomic molecule, $s$ tetrazine (ST). ST has been the subject of extensive spectroscopic studies over the past decade. ${ }^{9-12}$ It represents a system of particular interest since the molecule dissociates into $\mathbf{N}_{2}$ and $2 \mathrm{HCN}$ after excitation into the $\tilde{A}\left({ }^{1} B_{3 u}\right)$ state. ${ }^{13,14}$ This may cause some pecularities of the saturation experiment to be discussed in this work. Even though the quantum yield for dissociation is close to unity, ${ }^{15,16}$ the $\tilde{A}\left({ }^{1} B_{3 u}\right) \leftarrow \tilde{X}\left({ }^{1} A_{g}\right)$ spectrum reveals rotational fine structure and a Dopplerfree high resolution measurement is sensible. The dissociation of this molecule together with its sharp spectrum is a precondition for wavelength-selective excitation and dissociation and in particular for isotope-selective excitation. Isotope separation experiments have been successfully performed in $s$-tetrazine in the solid state $^{14}$ and the gas phase. ${ }^{13,17}$ The Doppler-free saturation spectra of the present work provide new information about the spectroscopy and the predissociation dynamics of this molecule. A point of particular interest will be whether rotation influences the decay dynamics of $\tilde{A}\left({ }^{1} B_{3 u}\right)$ ST.

\section{EXPERIMENTAL}

The experimental setup used for the recording of the Doppler-free spectra is shown schematically in Fig. 1. It resembles a Hänsch-Bordé-type saturation spectrometer ${ }^{18}$ with a reference beam added for increased sensitivity.

The narrow band tunable $\mathrm{cw}$ light is produced by a standing wave single-mode dye laser (CR 599) operating with Rhodamin 110 dye. With $2.5 \mathrm{~W}$ of $\mathrm{Ar}^{+}$-laser pump light, the output of the dye laser is about $100 \mathrm{~mW}$ in the frequency range needed. The linewidth was determined to be about $5 \mathrm{MHz}$. The wavelength of the laser light was measured with a commercial wavemeter (Burleigh WA-20) to an accuracy of 1 part in $10^{6}$. For a more precise calibration of the spectra, the iodine absorption spectrum ${ }^{19}$ was recorded simultaneously. The mode structure of the laser was controlled continuously with a scanning Fabry-Perot interferometer whose output signal was transformed into computerreadable information with the help of a home-built time-to-amplitude converter. 


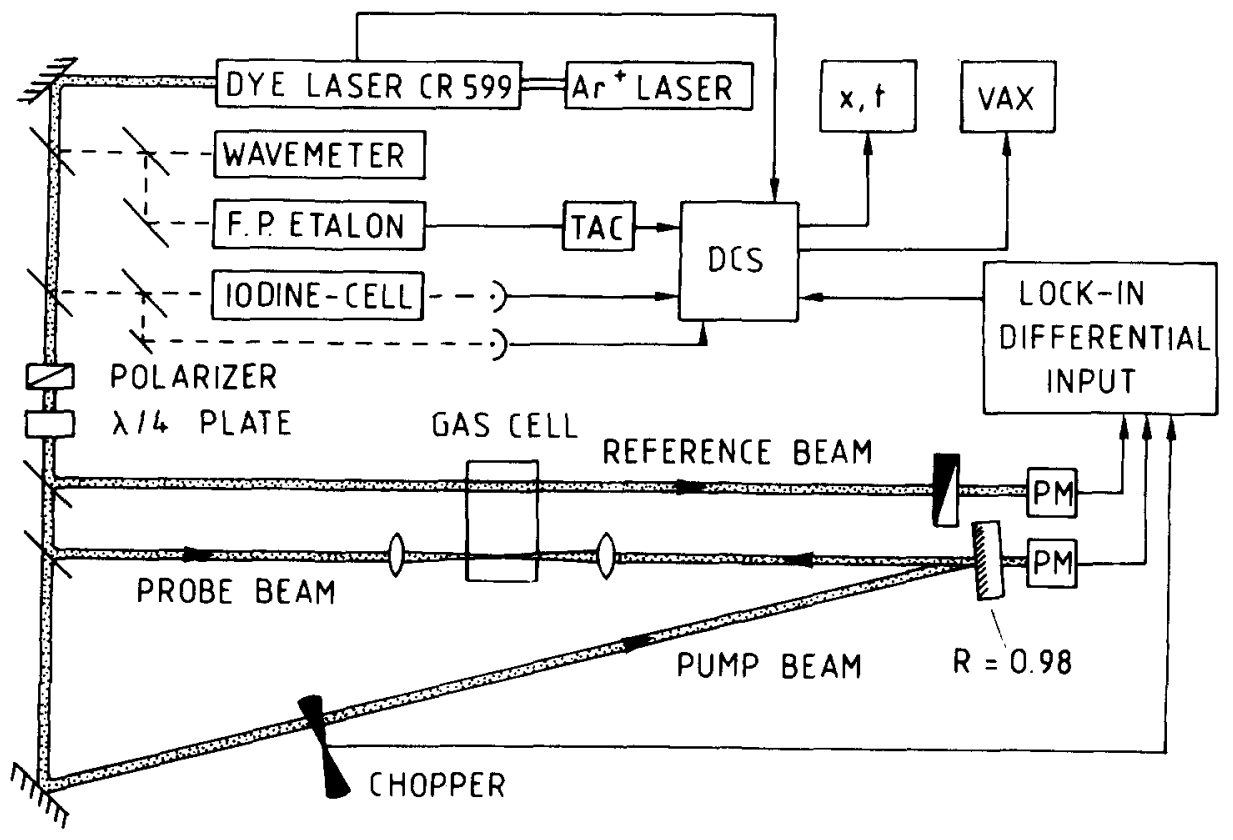

FIG. 1. Scheme of the experimental setup used for the measurement of Doppler-free saturation spectra of s-tetrazine.

The main part of the laser light is used as pump beam for the Doppler-free saturation experiment. The nonlinear interaction with the molecules (saturation) is detected as a decrease in absorption of a weak probe beam of the same frequency as the pump beam. As the pump and probe beam are counterpropagating and aligned collinearly, the two beams only interact with the same molecules if the laser frequency is equal to the transition frequency of molecules at rest and a Doppler-free spectrum is obtained. ${ }^{4,20}$ For the case of the $s$-tetrazine (ST) molecule, the "hole burning" (i.e., the selective depletion of molecules of one velocity group) is not simply the usual optical excitation of molecules but rather a photochemical process, as the ST molecules dissociate after excitation. For the experimental conditions used (i.e., weak absorption and small saturation) the linewidth observed in the spectrum is given by the homogeneous linewidth of the molecular transition. ${ }^{20}$ The saturation spectrum resembles an absorption spectrum without Doppler broadening. Additional "crossover" signals, which are frequently observed in saturation spectroscopy of atoms and small molecules, are not expected for the ST molecule due to the particular arrangement of transitions and the large spacing of rotational branches in the spectrum.

To suppress the Doppler-broadened lines in the spectrum, the pump beam is chopped at $2.3 \mathrm{KHz}$ and the probe beam signal, which is produced by the light transmitted through a mirror with $98 \%$ reflectivity, is evaluated in a lock-in amplifier. To increase the sensitivity of the setup, the varying amount of unmodulated signal at the lock-in was eliminated by the use of a weak reference beam which is also passed through the gas cell and substraction of the reference and probe signal in the lock-in. Both the probe and the reference beam are detected with matched 1 P28 photomultipliers and the output levels are carefully adjusted to equal value by adjusting the intensity of the reference beam. To avoid optical feedback into the dye laser, the experiment is decoupled by a combination of polarizer and $\lambda / 4$ retardation plate. In this way the light is circularly polarized in the gas cell.

The experimental setup was tested by recording the hyperfine spectrum of iodine. It was found that spectra could still be recorded with a ratio of $4 \times 10^{-5}$ between nonlinear ac signal and dc background. For the experiments on ST the pump and probe beam were focused into the gas cell $(17 \mathrm{~mm}$ path length) with lenses of $f=160 \mathrm{~mm}$. This allowed us to reach the intensity levels necessary for the partial saturation of the transitions of the molecule. Furthermore the cross section of the interaction region is drastically reduced by focusing of the beams. This proved necessary because of the photochemical instability of the ST molecule. For a stable molecule excited molecules return to the ground state via radiative and nonradiative decay and collisional deactivation. The photoexcited ST molecule on the contrary photodissociates with a probability near unity. ${ }^{15,16}$ This means that the molecules are actually destroyed so that the sample can be permanently bleached out in the interaction region. New ground state molecules will only be supplied by diffusion of ST into the illuminated region. This process is much faster for a small interaction region. To maintain a constant density of ST, a gas cell with a large volume was used. The gas density was monitored by the absorption of the laser light and the total pressure in the cell, as a large amount of dissociation of ST molecules into three product molecules (2 $\mathrm{HCN}+\mathrm{N}_{2}$ ) would result in an increase in the total pressure. The Doppler-free spectra and the calibration data were stored in a home-built DCS lab computer and displayed on a $x, t$ plotter for on-line control. Consequently the data were transferred to a VAX 11/780 for further processing and evaluation.

ST was prepared according to the method of Spencer $e t$ $a .^{21}$ and cleaned by multiple sublimation in order to avoid undesired contribution of water in the gaseous sample. Before filling the cell, the sample was carefully degassed. The cell was filled with substance in excess of the amount actually needed and the desired vapor pressure adjusted by keeping 


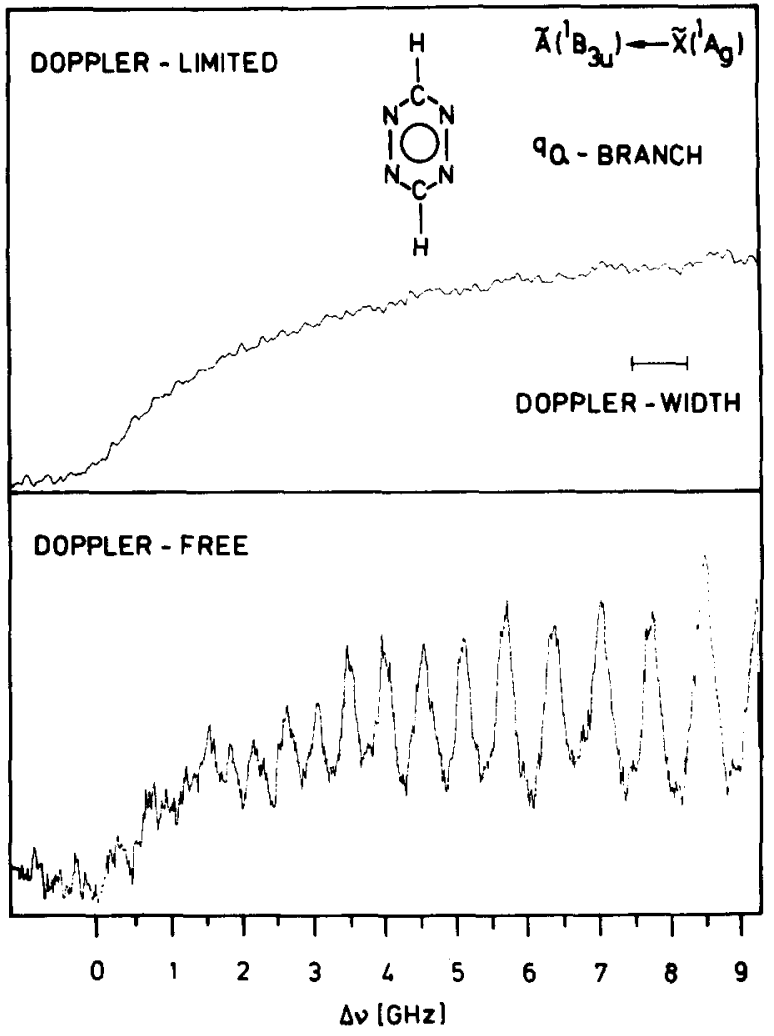

FIG. 2. Red edge of the ${ }^{q} Q$ branch of the $0-0$ band in s-tetrazine under Doppler-limited resolution (top). The same part of the spectrum measured with Doppler-free saturation spectroscopy (bottom).

a cold finger at appropriate temperature. The total pressure in the cell was measured with a capacitance manometer.

\section{RESULTS}

\section{A. Spectral analysis}

In Fig. 2 a small portion of the ${ }^{q} Q$ branch of the $0-0$ band is shown under Doppler-limited (top) and Dopplerfree (bottom) resolution. The spectrum represents the red edge of the ${ }^{q} Q$ branch and consists of rotational lines with low $J$ and $K_{c}$ values. As usual, $J$ is the quantum number of the total angular momentum and $K_{c}$ is the quantum number $K$ of its projection on the figure axis for the limiting oblate symmetric rotor. The Doppler-limited spectrum does not reveal a rotational fine structure within $10 \mathrm{GHz}$ to the blue from the rotational origin. This is due to the Doppler width of $720 \mathrm{MHz}$ for the transition frequency of about 18128 $\mathrm{cm}^{-1}$. On the other hand, a periodic rotational fine structure is clearly resolved in the Doppler-free spectrum. Figure 2 illustrates the increase of spectral resolution obtained when the nonlinear signal is observed in the saturation experiment as compared to the Doppler-limited signal.

In Fig. 3 the first $3.3 \mathrm{~cm}^{-1}$ of the Doppler-free spectrum of the ${ }^{q} Q$ branch of the $0-0$ transition are shown (middle trace). For comparison a calculated spectrum is plotted at the bottom of Fig. 3. The spectrum was calculated with an asymmetric rotor program for a parallel ( $C$-type $)^{9}$ transition. The rotational constants $A^{\prime \prime}, A^{\prime}, B^{\prime \prime}, B^{\prime}, C^{\prime \prime}$ were used as given by Job and Innes. ${ }^{10}$ The value $C^{\prime}=0.109785 \mathrm{~cm}^{-1}$ chosen for the calculation of the spectrum in Fig. 3 is very close to the value $C^{\prime}=0.10979 \mathrm{~cm}^{-1}$ of Ref. 10 . It has been found to yield a better fit of the periodic peak positions (see below ). All rotational constants are summarized in Table I. The calculated line spectrum was convoluted with a Lorentzian linewidth of $190 \mathrm{MHz}$. This linewidth gives the best fit of the experimentally found linewidth (for a detailed discussion see below). The zero point of the frequency scale in Fig. 3 is the rotationless transition frequency $\left(v_{00}=18128.035\right.$ $\mathrm{cm}^{-1}$ ). It has been found from a comparison of calculated and measured peak positions in the first $40 \mathrm{GHz}$ of the spectrum. Calibration of absolute frequency is performed by comparison with the simultaneously scanned iodine absorption spectrum ${ }^{19}$ (top of Fig. 3). Linearity of the frequency scale during the laser scan is monitored with a $1.5 \mathrm{GHz}$ free spectral range interferometer (periodic structure at the extreme top of Fig. 3).

The most striking feature of the spectrum in Fig. 3 is the periodic structure of the ${ }^{q} Q$ branch. ${ }^{11}$ The distance between neighboring peaks increases from $400 \mathrm{MHz}$ at the red edge of the ${ }^{q} Q$ branch to about $2.7 \mathrm{GHz}$ on the blue side of the spectrum shown in Fig. 3. As is seen in Fig. 2, in a Dopplerlimited spectrum the periodic structure is not resolved at the

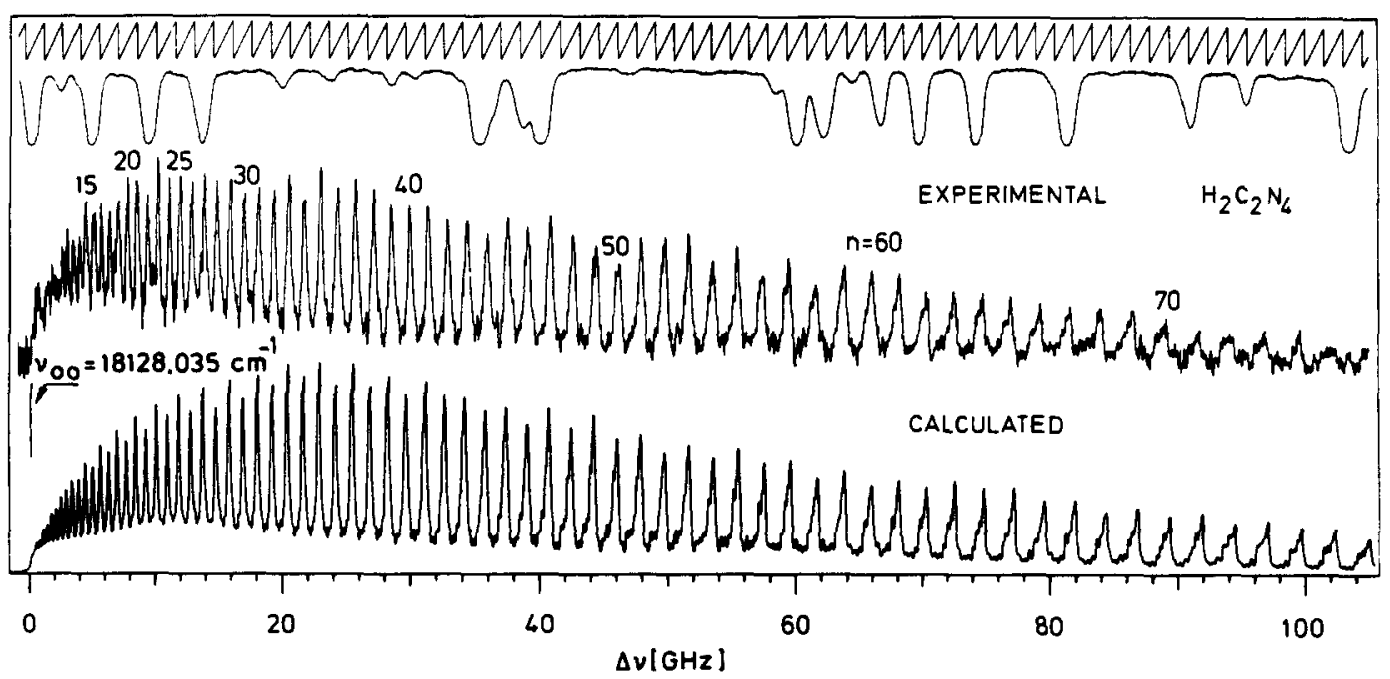

FIG. 3. Doppler-free saturation spectrum of the ${ }^{q} Q$ branch of the $0-0$ band in the $\tilde{A}\left({ }^{1} B_{3 u}\right)-\tilde{X}\left({ }^{1} A_{g}\right)$ system of $s$-tetrazine (middle trace). The calculated spectrum (lower trace) was convoluted with a Lorentzian linewidth of $\gamma=190 \mathrm{MHz}$. For frequency calibration the iodine absorption spectrum and the signal from an interferometer are shown at the top. 
TABLE I. Rotational constants of the s-tetrazine molecule used to reproduce the experimental spectrum. The constants were taken from Ref. 10 except $C^{\prime}$, which was determined in this work.

\begin{tabular}{ll}
\hline \hline Ground state $\widetilde{X}\left({ }^{1} A_{g}\right)$ & Excited state $\widetilde{A}\left({ }^{1} B_{3 u}\right)$ \\
\hline$A^{\prime \prime}=0.22696\left(\mathrm{~cm}^{-1}\right)$ & $A^{\prime}=0.22195\left(\mathrm{~cm}^{-1}\right)$ \\
$B^{\prime \prime}=0.21025$ & $B^{\prime}=0.21718$ \\
$C^{\prime \prime}=0.1092$ & $C^{\prime}=0.109785$ \\
\hline
\end{tabular}

red edge of the ${ }^{q} Q$ branch. In this spectrum the peaks can only be resolved at an energy of more than $12-14 \mathrm{GHz}$ to the blue of the rotationless origin, i.e., when the distance of neighboring peaks has increased to at least $1 \mathrm{GHz}$. The origin of the periodic peaks in the spectrum is explained by the Fortrat analysis of part of the ${ }^{q} Q$ branch shown in Fig. 4. It represents the result of an asymmetric rotor calculation. Only those few peaks which are located close to the rotationless origin of the ${ }^{q} Q$ branch (see below) result from single rotational transitions. All other peaks consist of many rotational lines which accumulate at the peak maximum. The peak maximum is given by lines with $K_{c} \leqslant J$. Using Gora's formula ${ }^{22}$ the position of the ${ }^{q} Q$ branch lines of a nearly oblate symmetric top can be approximated by

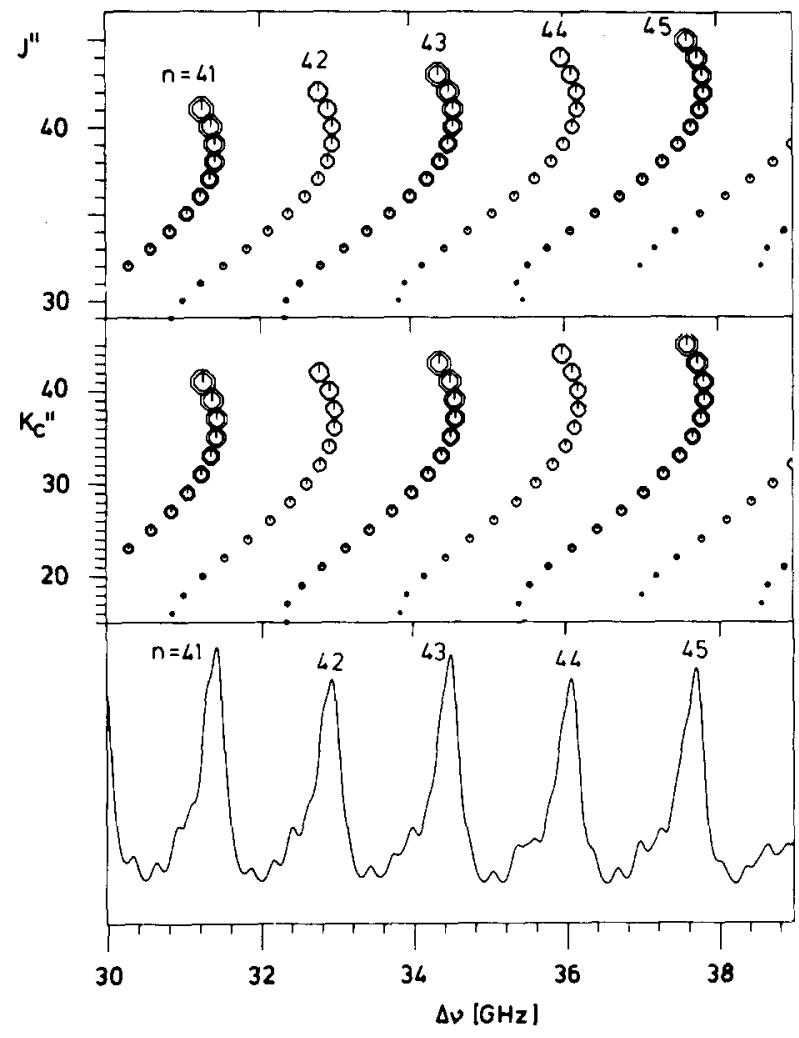

FIG. 4. Fortrat analysis (upper and middle plot) of a small part of the ${ }^{q} Q$ branch between 30 and $39 \mathrm{GHz}$ to the blue of the rotationless origin of the $0-0$ band in the $\tilde{A}\left({ }^{1} B_{3 u}\right) \leftarrow \tilde{X}\left({ }^{1} A_{8}\right)$ system of $s$-tetrazine. Positions of rotational transitions with different $J^{\prime \prime}$ (top) and $K_{c}^{\prime \prime}$ (middle) are given as a function of energy difference from the rotational origin. The area of the circles represents transition strengths. Due to the ordering of the rotational transitions, lines with particular $J^{\prime \prime}$ and $K_{c}^{\prime \prime}$ form a peak in the spectrum which is characterized by a pseudoquantum number $n$ according to Ref. 22 [see Eq. (4) of this work].

$$
\begin{aligned}
E= & E_{0}+(n+1)^{2}\left(C^{\prime}-C^{\prime \prime}\right) \\
& -1 / 4\left(2 m^{2}+2 m+1\right)\left(A^{\prime}-A^{\prime \prime}+B^{\prime}-B^{\prime \prime}\right) .
\end{aligned}
$$

Here

$$
n=2 J-K_{c}
$$

and

$$
m=J-K_{c},
$$

where $J=J^{\prime}, J^{\prime \prime}$ and $K_{c}=K_{c}^{\prime}, K_{c}^{\prime \prime} \cdot E_{0}$ is the energy of the rotationless transition. Since the strongest lines are those with $K_{c} \lesssim J$ we may neglect the $m$ dependent term in Eq. (1) and the peak positions in the spectrum of Fig. 3 are given by $^{23}$

$E-E_{0}=(n+1)^{2}\left(C^{\prime}-C^{\prime \prime}\right)$ with $n=1,2,3, \ldots$

For this reason the peaks in Fig. 3 can be characterized by the pseudoquantum number $n$. It is interesting to note that peaks with $n$ even are made up solely from rotational transitions with $K_{c}$ even and peaks with $n$ odd from $K_{c}$ odd. This is also demonstrated in the Fortrat analysis of Fig. 4 which represents the exact solution of the asymmetric rotor problem. Hence, the rotational transitions in the ${ }^{q} Q$ branch are sorted according to the symmetry of the ground state rotational wave function. $n$ even corresponds to rotational states with $K_{a}^{\prime \prime}, K_{c}^{\prime \prime}=$ oe,ee, ${ }^{24}$ whereas peaks with $n$ odd contain transitions starting from eo, oo rotational states. The corresponding symmetries of the nuclear spin wave functions are $B_{3}$ (ee), $A_{1}(\mathrm{eo}), B_{1}(\mathrm{oo})$, and $B_{2}$ (oe) and the statistical weights 9 (ee), 8 (eo), $11(\mathrm{oo})$, and $8(\mathrm{oe}) .^{25}$ The total statistical weight of $n$ even peaks is 17 and that of $n$ odd peaks is 19 . This gives rise to the alternating intensity of the peaks with $n>15$ clearly seen in the calculated spectrum of Fig. 3 and Fig. 4. In some parts of the experimental spectrum (Fig. 3) with good signal-to-noise ratio the intensity ratio is well reproduced.

From the energy formula in Eq. (4) and the measured spectrum, a value for $C^{\prime}-C^{\prime \prime}$ can be determined. We find a value $C^{\prime}-C^{\prime \prime}=0.000585 \pm 0.000006 \mathrm{~cm}^{-1}$ for peaks with $n<60$. This value agrees very well with the value previously found by Brown ${ }^{23}$ with the same approximation. It is close to the value of $\Delta C=C^{\prime}-C^{\prime \prime}$ found by Job and Innes ${ }^{10}$ from a complete asymmetric rotor analysis of this band. The error of $0.000006 \mathrm{~cm}^{-1}$ mainly reflects the experimental uncertainty in the calibration of the frequency scale, and it does not take into account the uncertainty due to the approximations in Eq. (4). In order to rule out these uncertainties, we performed an exact asymmetric rotor calculation of the spectrum with the value of $C^{\prime}-C^{\prime \prime}=0.000585 \mathrm{~cm}^{-1}$ found from Gora's approximation. The resulting calculated spectrum was convoluted with a Lorentzian linewidth of 190 $\mathrm{MHz}$ (FWHM) and is shown at the bottom of Fig. 3. Peak positions as well as peak structures $(n>50)$ are found to be in very good agreement with the measured spectrum.

\section{B. Homogeneous linewidths}

So far the organization of the spectrum and the information about the rotational constants have been discussed. Both are important preconditions for the determination of homogeneous linewidths and their interpretation. 


\section{Power broadening and collisional broadening}

The best simulation of the experimental spectrum in Fig. 3 was obtained by convolution of the calculated spectrum with a Lorentzian linewidth of $190 \mathrm{MHz}$. This corresponds to a three- to fourfold improvement of the effective spectral resolution as compared to that of Doppler-limited spectroscopy. Obviously the spectral resolution achieved in the Doppler-free spectrum is not the resolution expected from the frequency width ( $5 \mathrm{MHz}$ ) of the dye laser. Thus it is clear that the spectral resolution is not limited by the frequency width of the laser light and several other sources limiting the effective resolution have to be discussed.

One of the intrinsic broadening mechanisms in saturation spectroscopy is power broadening. ${ }^{26}$ It results fròm a shortening of the lifetime of the excited state due to stimulated emission from this state. In order to avoid power broadening the light intensity has to be smaller than the saturation intensity $I_{\text {sat }}$. The saturation intensity of the $s$-tetrazine molecule was measured in a separate experiment without focusing of the pump and the probe beam. The overlap of both light beams was carefully adjusted by observation of the visible fluorescence of ST and the intensity of the pump beam was chosen much smaller than the saturation intensity $I_{\text {sat }}$. The saturation intensity of the $\tilde{A} \leftarrow \tilde{X}$ band of ST is larger by nearly two orders of magnitude than $I_{\text {sat }}$ in the well-studied transition of iodine $\left(I_{\mathrm{sat}}=6 \mathrm{~W} / \mathrm{cm}^{2}{ }^{7}\right)$. For the $0-0$ band, a value of $I_{\text {sat }}=200 \mathrm{~W} / \mathrm{cm}^{2}$ was found. Since the absorption coefficient of both molecules is not very different, we conclude that this difference is caused by the much shorter lifetime of the excited state in ST. The Doppler-free spectrum of the $0-0$ band (see Fig. 3) was measured with a pump beam intensity of less than $40 \mathrm{~W} / \mathrm{cm}^{2}$ which is smaller by nearly one order of magnitude than $I_{\text {sat }}$. For this reason we expect a very small power broadening of about $2 \%$ which certainly does not contribute very much to the observed broadening in the spectrum. The Doppler-free spectrum of the $16 a_{1}^{1}$ band (see below) was measured with a higher laser intensity. The power broadening is estimated to be about $8 \%$ in this case. After having excluded power broadening as the source of the observed broadening, the influence of collisional broadening has to be checked. The spectrum of the red edge of the ${ }^{q} Q$ branch was measured for various pressures of ST below 1.5 Torr. The theoretical spectrum was then convoluted with various Lorentzian linewidths to determine which broadening yields the best fit to each experimental spectrum according to the procedure described below. The linewidths thus obtained are plotted in Fig. 5 for pressures ranging from 0.1 to 1.4 Torr. In the observed pressure range, the pressure dependence of the linewidth can be fitted by a straight line and the collisionless linewidth is found in the usual way from an extrapolation of the straight line to zero pressure. Obviously at the lowest pressure of 0.1 Torr, the contribution of collisional broadening to the measured linewidth is much smaller than the experimental error and may be neglected.

The slope of the straight line in Fig. 5 yields the collisional line broadening parameter. We find an exceptionally large value of $\gamma_{\text {coll }}=290 \pm 30 \mathrm{MHz} /$ Torr. This is by more than one order of magnitude larger than the collisional line broadening parameter $\left(\gamma_{\text {coll }}=15 \mathrm{MHz} / \mathrm{Torr}\right)$ of $\mathrm{C}_{6} \mathrm{H}_{6}$

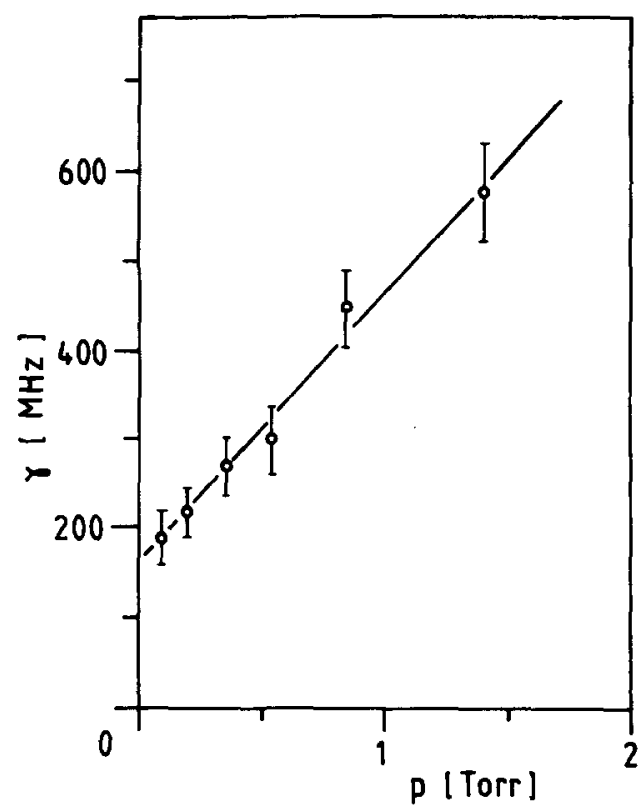

FIG. 5. Measured pressure dependence of the homogeneous linewidth in the $0-0$ band of $s$-tetrazine in the pressure range between 0.1 and 1.4 Torr. The solid line represents the best linear fit to the experimental points. Error bars indicate the accuracy of the procedure for the determination of the homogeneous linewidth.

found in our previous ultrahigh resolution Doppler-free two-photon experiment. ${ }^{3}$ Furthermore it is also larger by one order of magnitude than the cross sections found for rotational relaxation in benzene. ${ }^{27}$ The corresponding collisional rate constant $k_{\text {coll }}=2 \pi \gamma_{\text {coll }}=1.8 \times 10^{9} \mathrm{~s}^{-1}$ Torr $^{-1}$ cannot be explained by gas kinetic hard sphere collisions at room temperature which would lead to a value smaller by two orders of magnitude. Possibly the highly efficient ( $99 \%$ quantum yield) dissociation of ST after excitation to the $S_{1}$ state results in additional line broadening effects. Even though the reason for the extraordinarily large collisional line broadening parameter (steep slope of the Stern-Vollmer plot in Fig. 5) is not clear at present time, the extrapolation of linewidth to zero pressure is reliable. Since the collisional line broadening parameter is very large already at pressures above $100 \mathrm{mTorr}$, a further increase at lower pressure is hardly expected.

From the above discussion it is evident that there is no remarkable contribution to the measured linewidth either from power broadening or from collisions at the low pressure of $100 \mathrm{mT}$ Trr. The linewidth found from a simulation of the spectrum is therefore the actual collisionless homogeneous linewidth.

\section{O-O band: Rotatlonal dependence of linewidth}

For detailed analysis of the homogeneous intramolecular linewidth a magnified portion of the edge of the ${ }^{9} Q$ branch is shown at the top of Fig. 6. It was measured with the sub-Doppler resolution of the saturation spectroscopy experiment. The focusing conditions (intensity, beam diameter) were optimized for this scan so that the signal to noise ratio is somewhat better than in the spectrum of Fig. 2. At the bottom of Fig. 6 the calculated rotational line spectrum is 


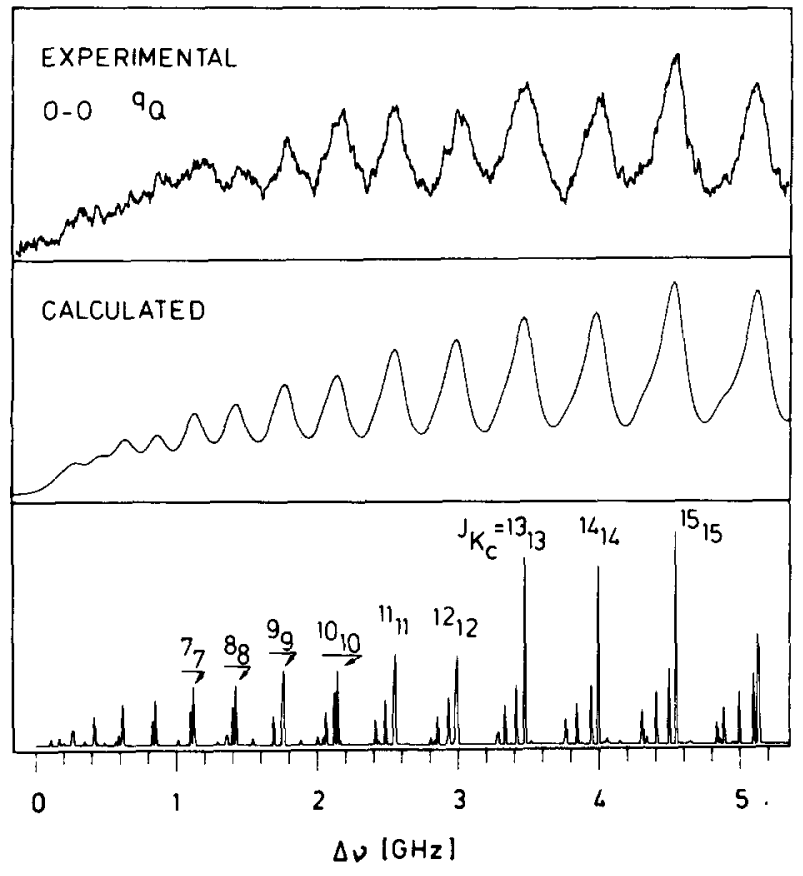

FIG. 6. Red edge of the ${ }^{q} Q$ branch of the $\tilde{A}\left({ }^{1} B_{3 u}\right)-\tilde{X}\left({ }^{1} A_{g}\right)$ transition of $s$ tetrazine. Top: Measured Doppler-free saturation spectrum. Middle: Calculated spectrum obtained by convolution of the stick spectrum (bottom) with a Lorentzian linewidth of $\gamma=190 \mathrm{MHz}$. This linewidth gives the best simulation of the measured spectrum. Bottorn: Calculated stick spectrum. For the strongest lines the $J, K_{c}$ quantum numbers are given.

shown. The convolution of this spectrum with a Lorentzian linewidth of $190 \mathrm{MHz}$ leads to the spectrum in the middle trace. This linewidth leads to the best reproduction of the measured spectrum. In order to rule out errors due to an inaccurate determination of the rotational constants which might simulate a wrong linewidth we performed an additional test. From the theoretical stick spectrum (bottom trace of Fig. 6), it is seen that for the strongest lines $K_{c}=J$ and that a periodic structure arises from the particular grouping of the rotational lines. The periodic peaks shown in the measured spectrum (top) and the convoluted spectrum (middle) are due to this grouping and do not represent single rotational lines. The maxima are given by the strong $K_{c}=J$ transitions and they are red shaded due to additional weaker rotational lines with particular quantum numbers (see Sec. III A). Each peak has a sharp edge on the blue side which is mainly determined by the shape of the strongest line, i.e., the $K_{c}=J$ line. The wings of the peaks do not reach zero; there is a broad background underlying the peaks due to the overlapping wings of all rotational lines with Lorentzian shape. We found that the peak height to background ratio is very sensitive to the width of the Lorentzian line shape. From this we determined the homogeneous linewidth of the rotational transitions. From an analysis of all peaks from $J_{K_{c}}=8_{8}$ to $15_{15}$ we find a value of $\gamma=190 \pm 15 \mathrm{MHz}$ for the Lorentzian linewidth which shows no dependence on the rotational quantum number within the experimental accuracy.

For peaks with increasing pseudoquantum number the situation is more complicated since for $n>15$ rotational lines with $K_{c}<J$ contribute more strongly to the peaks. An estimate can be obtained from the comparison of the Doppler-free spectrum with calculated spectra of different width. The best agreement is obtained for all values of $n$ for a Lorentzian linewidth of $\gamma=190 \mathrm{MHz}$. No difference in the linewidth can be found with increasing $n$, the complete spectrum up to $J=70$ can be fitted with a single Lorentzian linewidth of $\gamma=190 \mathrm{MHz}$. From this it is concluded that there exists no $J$ dependence of the homogeneous linewidth. Hence the intramolecular relaxation rate responsible for the linewidth is also independent of the rotational quantum number.

\section{3. $16 a_{1}^{7}$ band}

In Fig. 7 the red edge of the ${ }^{q} Q$ branch of the $0-0$ transition (bottom) ( $\left.v_{00}=18128.035 \mathrm{~cm}^{-1}\right)$ is compared with the corresponding part of the $16 a_{1}^{1}$ vibronic band $\left(v_{00}=18047.70 \mathrm{~cm}^{-1}\right)$ (top). The $16 a_{1}^{1}$ band $\left(v_{16 a}^{\prime}=256\right.$ $\mathrm{cm}^{-1}$ ) is a sequence band of the $0-0$ band. It also results from parallel transitions. For the low $J, K_{c}$ values observed at the edge of the ${ }^{q} Q$ branch, the small differences in the rotational constants expected for the two bands would not lead to pronounced changes in the rotational structure. We can directly use the rotational analysis of the $0-0$ band (Fig. 6) for the determination of the homogeneous linewidth in the $16 a_{1}^{1}$ band. From a comparison of both spectra in Fig. 7 it is seen that the rotational structure of the $16 a_{1}^{1}$ band is resolved for even smaller $J$ values than in the $0-0$ band. Even

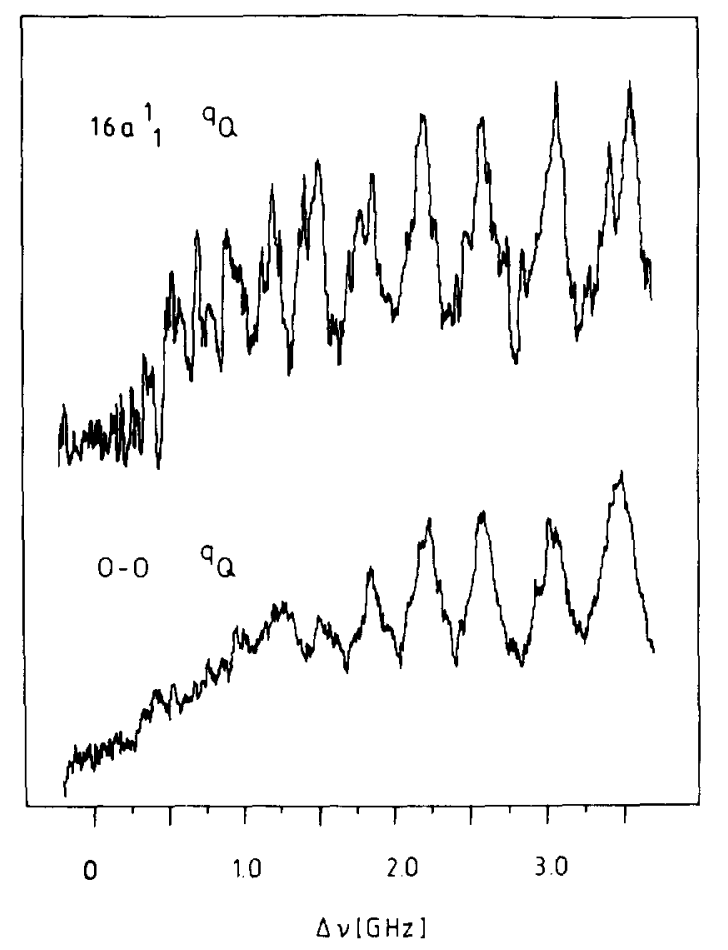

FIG. 7. Comparison of measured Doppler-free saturation spectra of corresponding parts of two vibronic bands in the $\vec{A}\left({ }^{1} B_{3 u}\right)-\tilde{X}\left({ }^{1} A_{g}\right)$ system of $s$-tetrazine. Upper trace: Red edge of the ${ }^{q} Q$ branch of the $16 a_{1}^{1}$ band. Lower trace: Red edge of the ${ }^{9} Q$ branch of the $0-0$ band. 
TABLE II. Rotationless transition frequencies and homogeneous linewidths of the $0-0$ and $16 a_{1}^{1}$ bands of $s$-tetrazine as determined in this work. The relaxation rates and corresponding lifetimes which can be deduced from the linewidth are given for comparison with the previously measured values (Refs. 12 and 28).

\begin{tabular}{llllrrr}
\hline \hline Transition & $v\left(\mathrm{~cm}^{-1}\right)$ & $\gamma(\mathrm{MHz})$ & $k\left(\mathrm{~s}^{-1}\right)$ & $\tau(\mathrm{ps})$ & $\tau(\mathrm{ps})^{\mathrm{a}}$ & $\tau(\mathrm{ps})^{\mathrm{b}}$ \\
\hline $0-0$ & 18128.035 & $190 \pm 15$ & $1.19 \times 10^{9}$ & 838 & 820 & 700 \\
$16 a_{1}^{1}$ & 18047.70 & $140 \pm 50$ & $0.88 \times 10^{9}$ & 1137 & 1470 & \\
\hline
\end{tabular}

${ }^{\mathrm{a}}$ From Ref. 28.

${ }^{b}$ From Ref. 12.

though the spectrum is somewhat noisy, it is clear that the homogeneous linewidth in the $16 a_{1}^{1}$ band is smaller than in the $0-0$ band. The procedure described above now yields $\gamma=140 \pm 50 \mathrm{MHz}$. Both values of the intramolecular relaxation linewidth are summarized in Table II together with the corresponding inferred relaxation rates $k=2 \pi \gamma$.

\section{DISCUSSION AND CONCLUSION}

In this work we have shown that Doppler-free saturation spectroscopy is feasible on large molecules. As a first example, Doppler-free spectra of two bands in the $\tilde{A}\left({ }^{1} B_{3 u}\right) \leftarrow \tilde{X}\left({ }^{1} A_{g}\right)$ transition of $s$-tetrazine have been measured. At the red edge of the ${ }^{q} Q$ branch the periodic rotational microstructure is resolved down to smaller pseudoquantum numbers $(n=8)$ than in conventional high resolution spectroscopy $(n=23)$. From a computer simulation of the periodic structure a precise value for the change of the rotational constant $C$ (i.e., $\Delta C=C^{\prime}-C^{\prime \prime}$ ) is obtained. This value confirms previous results from Doppler-limited spectra. ${ }^{10,23}$

Homogeneous linewidths have been measured for the $0-0$ band and the $16 a_{1}^{1}$ vibronic transition leading to the $16 a^{1}$ vibrational level at $256 \mathrm{~cm}^{-1}$ excess energy. The homogeneous linewidth was found to decrease from $190 \pm 15 \mathrm{MHz}$ for the $0-0$ transition to $140 \pm 50 \mathrm{MHz}$ for the $16 a_{1}^{1}$ band. Even though our results have been obtained by a completely different method they compare quite well with recent lifetime measurements of these bands by Langelaar et al. ${ }^{28} \mathrm{Life}-$ time results as well as the decay rates deduced from recent linewidth measurements for the $0-0$ band from supersonic beam measurements ${ }^{12}$ are summarized in Table II. The somewhat larger linewidth in the beam experiment may be explained by power broadening as indicated by the authors.

It is interesting to see that the relaxation rate $\left(0.88 \times 10^{9}\right.$ $\mathrm{s}^{-1}$ ) for the $16 a^{1}$ state with an excess energy of $256 \mathrm{~cm}^{-1}$ is smaller than that of the vibrationless level of the $\tilde{A}\left({ }^{1} B_{3 u}\right)$ state. Most likely the measured relaxation rate represents the rate of a photophysical primary process (electronic nonradiative process) prior to dissociation. Nonradiative processes have been found to be dependent on the nature of the vibrational modes in organic molecules of similar size, e.g., in pyrazine ${ }^{29}$ and benzene. ${ }^{30,31}$ There the electronic nonradiative process is influenced not only by electronic matrix elements but also by Franck-Condon factors which might lead to the observed vibrational dependence of the electronic nonradiative relaxation rate.

So far we have discussed the vibrational dependence of the measured decay rate. This led to the conclusion that the measured decay rate is that of a primary electronic nonradiative process. Now the additional experimental information about the rotational dependence, presented for the first time in this work, has to be discussed. Several attempts have been made to study the influence of rotation on the decay of electronically excited ST. Aartsma $e t a l .^{32}$ measured the resonant CARS spectrum of the $1008 \mathrm{~cm}^{-1}$ vibration and found a shift of the intensity maximum towards the red edge of the ${ }^{q} Q$ branch. For a possible explanation of this shift they invoked an increase of electronic transition homogeneous linewidth with increasing rotational quantum number. The varying linewidth was thought to be caused by a rotationally dependent dissociation or a rotationally dependent nonradiative process. Picosecond lifetime measurements were performed by Langelaar et al. ${ }^{28}$ The bandwidth of their picosecond laser was $60 \mathrm{GHz}$ and did not allow for selective excitation of single rotational lines within the $0-0$ band. They measured the decay rate as a function of the excitation energy difference with respect to the central ${ }^{q} Q$ branch. The $20 \%$ change in decay rate was interpreted as due to an increase of decay rate with rotational energy. In contrast to these results the detailed spectroscopic study and the homogeneous linewidth measurements in a cooled supersonic beam of Brumbaugh et al. ${ }^{12}$ did not show a rotational effect on linewidth up to $J=4$. Obviously, a rotational dependence of the relaxation rate is not observed in our experiment. Theory of nonradiative transitions ${ }^{33}$ predicts no rotational dependence of a nonradiative decay if it is in the statistical limit. This has indeed been experimentally verified in our recent work for the molecule benzene. ${ }^{34}$ Single rotational level lifetime measurements after pulsed Doppler-free two-photon excitation revealed no rotational dependence of the electronic nonradiative decay rate which is probably intersystem crossing (ISC) in the statistical limit at the excess energy of this experiment. This result very much resembles the situation present in $s$-tetrazine shown in this work. We again conclude that the observed decay rate is that of an electronic nonradiative process in the statistical limit. In addition, from the lack of rotational effects and the small $S_{1}-T_{1}$ energy gap $\left(4520 \mathrm{~cm}^{-1}\right)^{36}$ in s-tetrazine it can be argued that the nonradiative process is more likely internal conversion (IC) ${ }^{35}$ than ISC. The absorption of a second photon before dissociation, which has been assumed in the pulsed experiments of Ref. 37, can be excluded for the low cw light intensities of the present experiment and our previous isotope-selective photodissociaton. ${ }^{17}$ IC to the $\tilde{X}\left({ }^{1} A_{g}\right)$ state is then assumed to be a primary process to the dissociation of 
ST. From this we may conclude that $s$-tetrazine dissociates according to a RRKM statistical type of dissociation on the ground state potential curve rather than by a dissociation from a repulsive potential curve.

In conclusion we have shown that Doppler-free high resolution spectroscopy provides valuable information on the dynamics of photodissociation. Our results demonstrate the importance of rate determining primary nonradiative processes for the photodissociation of polyatomic molecules.

\section{ACKNOWLEDGMENTS}

The authors are indebted to Professor Schlag for his permanent interest in this work. They wish to thank Professor Parmenter for critical reading of the manuscript.

${ }^{1}$ E. Riedle, H. Stepp, and H. J. Neusser, Chem. Phys. Lett. 110, 452 (1984).

${ }^{2}$ E. Riedle, H. J. Neusser, and E. W. Schlag, J. Phys. Chem. 86, 4847 (1982).

${ }^{3}$ E. Riedle and H. J. Neusser, J. Chem. Phys. 80, 4686 (1984).

${ }^{4}$ For a review see W. Demtröder, Laser Spectroscopy (Springer, Berlin, 1981).

${ }^{5}$ W. M. McClain, J. Chem. Phys. 55, 2789 (1971).

${ }^{6}$ T. W. Hänsch, I. S. Shahin, and A. L. Schawlow, Phys. Rev. Lett. 27, 707 (1971).

${ }^{7}$ T. W. Hänsch, M. D. Levenson, and A. L. Schawlow, Phys. Rev. Lett. 26, 946 (1971)

${ }^{8}$ R. S. Lowe, H. Gerhardt, W. Dillenschneider, R. F. Curl, Jr., and F. K. Tittel, J. Chem. Phys. 70, 42 (1979); K. H. Fung and D. A. Ramsay, J. Phys. Chem. 88, 395 (1984).

${ }^{9}$ A. J. Merer and K. K. Innes, Proc. R. Soc. London Ser. A 302, 271 (1968).

${ }^{10}$ V. A. Job and K. K. Innes, J. Mol. Spectrosc. 71, 299 (1978).

${ }^{11}$ K. K. Innes, A. H. Kalantar, A. Y. Khan, and T. J. Durnick, J. Mol. Spectrosc. 43, 477 (1972).
${ }^{12}$ D. V. Brumbaugh, C. A. Haynam, and D. H. Levy, J. Mol. Spectrosc. 94, $316(1982)$.

${ }^{13}$ R. R. Karl and K. K. Innes, Chem. Phys. Lett. 36, 275 (1975).

${ }^{14}$ R. M. Hochstrasser and D. S. King, J. Am. Chem. Soc. 97, 4760 (1975).

${ }^{15}$ G. K. Vemulapalli and T. Cassen, J. Chem. Phys. 56, 5120 (1972).

${ }^{16}$ J. H. Meyling, R. P. van der Werf, and D. A. Wiersma, Chem. Phys. Lett. 28, 364 (1974).

${ }^{17}$ U. Boesl, H. J. Neusser, and E. W. Schlag, Chem. Phys. Lett. 61, 62 (1979).

${ }^{18}$ See, for example, M. D. Levenson, Introduction to Nonlinear Laser Spectroscopy (Academic, New York, 1982), p. 66ff.

${ }^{19}$ S. Gerstenkorn and P. Luc, Atlas du Spectred Absorption de la Molecule $d$ Iode (Edition du CNRS, Paris, 1978). The systematic error of $-0.0056 \mathrm{~cm}^{-1}$ found by S. Gerstenkorn and P. Luc [Rev. Phys. Appl. 14, 791 (1979] has not been taken into account in the present work.

${ }^{20}$ For a detailed discussion see, V. S. Letokhov and V. P. Chebotayev, Nonlinear Laser Spectroscopy, edited by D. L. MacAdam, Springer Series in Optical Science (Springer, Berlin, 1977), Vol. 4.

${ }^{21}$ G. H. Spencer, Jr., P. C. Cross, and K. B. Wiberg, J. Chem. Phys. 35, 1939 (1961).

${ }^{22}$ E. K. Gora, J. Mol. Spectrosc. 16, 378 (1965).

${ }^{23}$ J. M. Brown, Can. J. Phys. 47, 233 (1969).

${ }^{24}$ For definition of the symbols used, see, H. W. Kroto, Molecular Rotation Spectra (Wiley, London, 1975).

${ }^{25}$ A. Kiermeier, Diplomarbeit, Technische Universität München, 1983.

${ }^{26}$ V. S. Letokhov and V. P. Chebotayev, Nonlinear Laser Spectroscopy, Springer Series in Optical Sciences (Springer, Berlin, 1977), Vol. 4.

${ }^{27}$ C. S. Parmenter, B. Setzer, and K. Y. Tang, J. Chem. Phys. 66, 1317 (1977); R. A. Coveleskie and C. S. Parmenter, ibid. 69, 1044 (1978).

${ }^{28}$ J. Langelaar, D. Bebelaar, M. W. Leeuw, and J. J. F. Ramaekers, in Picosecond Phenomena II, edited by R. M. Hochstrasser, W. Kaiser, and C. V. Shank, Springer Series in Chemical Physics (Springer, Berlin, 1980), Vol. 14.

${ }^{29}$ A. E. W. Knight and C. S. Parmenter, Chem. Phys. 15, 85 (1976).

${ }^{30}$ K. G. Spears and S. A. Rice, J. Chem. Phys. 55, 5561 (1971).

${ }^{31}$ L. Wunsch, H. J. Neusser, and E. W. Schlag, Chem. Phys. Lett. 32, 210 (1975).

${ }^{32}$ T. J. Aartsma, W. H. Hesselink, and D. A. Wiersma, Chem. Phys. Lett. 71, $424(1980)$.

${ }^{33}$ F. A. Novak and S. A. Rice, J. Chem. Phys. 73, 858 (1980).

${ }^{34}$ U. Schubert, E. Riedle, and H. J. Neusser, J. Chem. Phys. 84, 5326 (1986).

35J. R. McDonald and L. E. Brus, J. Chem. Phys. 59, 4966 (1973).

${ }^{36}$ G. Fischer and G. J. Small, J. Chem. Phys. 56, 5934 (1972).

${ }^{37}$ J. H. Glownia and S. J. Riley, Chem. Phys. Lett. 71, 429 (1980). 